\title{
Lokale Strategien der (sprachlichen) Realisierung von globalen Feindbildern
}

Nikolina Palašić (Rijeka)

\begin{abstract}
The goal of this paper is to try to show how language can be used to construct hostile images and how on the basis of these images verbal and physical violence may arise. A recent situation in Croatia, which from present-day perspective can without reservation be described as an antigay campaign (2013), will serve as an example of hate speech. We use the statements and images that were used at that time in the media and by the general public to try and explain the origin of such words and images and to explain how they should be interpreted with respect to the Croatian social context.
\end{abstract}

\section{$1 \quad$ Einleitung oder Wenn Symbole zur Wirklichkeit werden}

Wir möchten hier Überlegungen zu der Frage aussparen, inwiefern der Kampf gegen verletzende Ausdrücke in einer durch Gesetze geregelten Gesellschaft im Gegensatz zur Redefreiheit steht, aber es soll nur erwähnt werden, dass es manchmal nicht leicht ist, eine strikte Linie zwischen der bloßen Meinungsäußerung und der Gewalt durch die Sprache zu ziehen. Wer verletzende Ausdrücke in ihrer nicht sehr expliziten Form benutzt (z. B. Die Ehe zwischen Mann und Frau ist eine Moralfrage), kann sich immer dadurch rechtfertigen, dies sei nur seine Meinung, und sich somit auf die Meinungsfreiheit berufen. Die Gewalt durch Sprache kann häufig nur dann sanktioniert werden, wenn sie von physischer Gewalt begleitet wird oder sich eventuell als Drohung klassifizieren lässt. Daher kommt es oft vor, dass abwertende Ausdrücke, die generell gegen eine gesellschaftliche Gruppe gerichtet sind, nicht bestraft werden können, sondern stillschweigend erlaubt werden. Dass aber Worte nicht nur Worte sind, haben schon zahlreiche soziologische, psycholinguistische und sogar neurowissenschaftliche Untersuchungen gezeigt. Wollen wir dabei im Bereich der linguistischen Betrachtungen bleiben, können wir von Austins Theorie der Performativität ausgehen, laut welcher manche verbalen Äußerungen mit dem Handeln gleichzusetzen sind (Austin 1979: 29). Auf die Theorie der Performativität stützend, fragt sich Butler, „ob Sprache uns verletzen könnte, wenn wir nicht in einem bestimmten Sinne ,sprachliche Wesen“ wären, die der Sprache bedürfen, um zu sein“. Sie kommt zum Schluss, dass unsere Empfindlichkeit gegen das Sprachliche darauf beruht, „dass es ihre (sprachliche) Bedingungen sind, die uns konstituieren“ (2006: 9). Butler geht also auch davon aus, dass die Sprache eine performative Macht hat bzw. dass sie in der Lage ist, eine Realität zu konstruieren und nicht nur etwas über diese Realität zu äußern. Dabei handelt es sich um die 
Ausübung einer symbolischen Macht, was Sprache zu einem wichtigen, ja sogar zentralen Instrument sozialer Herrschaft macht (Bourdieu 1990: 46).

Die Sprache sorgt nämlich für einen großen Teil unserer Erfahrungen und sie ist Träger unseres konzeptuellen Denkens. ${ }^{1}$ Am Beispiel der verletzenden Verbalisierungen kann man betrachten, wie man durch die Sprache eine Wirklichkeit produziert, die es außerhalb der Sprache, also in der natürlichen Welt, zuerst gar nicht gab, aber die dann zur Realität auf einer symbolischen Ebene menschlichen Verstandes zu existieren anfängt und sich letztendlich in der realen Welt widerspiegelt.

Hasssprache operiert auf einer symbolischen Ebene, und zwar so, dass man durch solche verbalen Ausdrücke bestimmte negativ geladene Bilder von einer sozialen Gruppe bildet, die dann, mit Worten von Lakoff und Wehling (2008) „auf leisen Sohlen ins Gehirn“ der Menschen schleichen und einen symbolischen Kode für Gewalt kreieren. Sprachliche Äußerungen konstruieren unterschiedliche Konzeptualisierungen, d. h. sie wecken die Assoziationen, die mit bestimmten gesellschaftlichen Gruppen verbunden werden, egal ob die assoziierten Eigenschaften der Realität entsprechen oder nicht.

Pierre Bourdieu (2001) hat in seinem Interview über die Konstruktion einer symbolischen Ökonomie des Geschlechterverhältnisses versucht, das soziale Schaffen der Unnatürlichkeit im Zusammenhang mit den Homosexuellen zu erklären. So sagt er, dass dieses Widernatürlich-Sein auf der Hauptteilung der Menschen in eine weibliche und männliche Natur beruht, wobei schon diese Einteilung mit einer Vielfalt der Symbole einhergeht, d. h. der männlichen Natur die Eigenschaften „,sexuell aktiv“, „,dominant“, „penetrierend“ und der weiblichen ,sexuell passiv“, „unterworfen“, ,penetrant“" zugeschrieben werden. Homosexuelle Männer werden feminisiert, weil sie eine sexuelle Beziehung vorziehen, die sonst den Frauen zukommt. Deswegen beschreibt man diese Beziehung als unnatürlich, d. h. als eine Erscheinung, deren Existenz der sozialen Hierarchie widerspricht. ,Der Homosexuelle überschreitet jene Grenzen, die die Römer sehr wohl kannten: wenn man die aktive Homosexualität mit einem Sklaven noch tolerieren konnte, war jede passive Beziehung mit einem Beherrschten absolut monströs“ (2001: 25). In diesem Zusammenhang behauptet Bourdieu weiter, dass die Geschlechterbeziehung immer eine Herrschaftsbeziehung ist, und diese Idee sei „tief im Unbewussten“ der Menschen verankert (ebd.).

Etwas Vergleichbares konnte man 2013 auch in Kroatien erleben, als man ausführlich darüber diskutiert hat, ob man einem homosexuellen Paar erlauben soll, ein Kind zu adoptieren. Damals hat es am häufigsten geheißen: „Zwei Männer sollen ein Mädchen erziehen? Das ist doch unnatürlich!“ Von einer Möglichkeit, dass zwei Frauen ein Kind, egal ob Mädchen oder Junge, erziehen, hat kaum jemand behauptet, es sei unnatürlich. Die Homosexualitätsfrage wurde doch in den meisten Fällen mit der Unnatürlichkeit einer Ehe, oder überhaupt einer Beziehung, zwi-

\footnotetext{
${ }^{1}$ Lakoff und Johnson (1980) sind in ihrem Werk Metaphors we live by von der These ausgegangen, dass das ganze menschliche Denken in Metaphern abläuft, die sie dann konzeptuelle Metaphern genannt haben, aber sich die Menschen dieser Metaphern zumeist nicht bewusst sind. Die Haupteigenschaft der konzeptuellen Metaphern lässt sich als Fähigkeit, einige Aspekte zu betonen und andere auszublenden, beschreiben. Laut Jäkel (2003: 21) besteht das konzeptuelle Verständnis der Metaphern ,(...) in der systematischen Verbindung zwischen zwei verschiedenen konzeptuellen Domänen, von denen die eine (x) als Zielbereich (target domain) und die andere (y) als Ursprungsbereich (source domain) der metaphorischen Übertragung (metaphorical mapping) fungiert".
} 
schen zwei Männern verknüpft. Das Problem, das Homosexualität in einer Gesellschaft darstellt, spricht auch Judith Butler an und erklärt, es habe seine Wurzeln in einer ,phallogozentrischen Gewalt" bzw. in einer durch männliche Natur dominierten sozialen Konstellation (Butler 1999). Dieser Phallo(go)zentrismus ${ }^{2}$ hat natürlich auch einen Ausschließungscharakter, und dieser handelt durch das Wecken bestimmter mentaler Bilder, die durch den verbalen oder nicht-verbalen Kode zustande kommen. Im verbalen Kode bedient man sich der Worte auf dieselbe Art, wie man sich in einem kriegerischen Kampf der Waffe bedient - im Krieg nutzt man die Waffe, um ein Stück eines Landes zu erobern, und im Kampf gegen den sozial konstruierten Feind benutzt man Worte, um ein Stück des Gehirns bei den Menschen zu erobern, die man zu den Gleichgesinnten machen will.

\section{Vom Verhältnis zwischen Sprechen und Denken}

Unsere Gedanken sind latente Worte, und umgekehrt, unsere Worte sind Abbildungen unserer Gedanken, aber auch unseres Unbewussten, das von der kulturellen Umgebung implizit geprägt wurde. Wir wissen nicht, dass uns etwas stört, solange wir es nicht erfahren. Wie Hetzel (2010: 9) sagt, „Erfahrungen konstruieren sich in Rede und Gegenrede“. Es handelt sich dabei also um einen geschlossenen Kreis.

Hasssprache darf man nicht isoliert von einer bestimmten Denkweise betrachten. Beispielsweise wird in der Psycholinguistik häufig die Frage gestellt, in welchem Verhältnis Denken und Sprechen zueinanderstehen bzw. ob es Gedanken jenseits der Sprache sowie Sprechen ohne jegliche Gedanken gibt. Wir können heutzutage sagen, dass es schwer beweisbar ist, ob man Sprechen und Denken so strikt voneinander abgrenzen kann, da uns einerseits schon von der Saphir-Whorf-Hypothese bekannt ist, dass Sprache das Denken formt. Andererseits sagen einige psycholinguistische Ansätze, die auf der behavioristischen Auffassung beruhen, „dass Denken nichts als lautloses Sprechen sei. Denken ist (...) ein durch sozialen Druck, durch Sozialisationsdruck, durch Erziehungsmaßnahmen allmählich internalisiertes Sprechen“ (Graumann 1972: 144).

Diese zweite These, die das Denken und Sprechen gleichsetzt, finden wir hier auch etwas übertrieben, schon aus dem Grunde, weil es dabei um zwei komplett unterschiedliche geistige Ebenen geht, die unterschiedlichen Gesetzlichkeiten folgen. Man kann nicht ernsthaft behaupten, dass das Sprechen einfach ein 1:1-Lautwerden dessen ist, was einem gerade durch den Kopf geht. Hier vertreten wir die These, dass Denken und Sprechen nicht eins und dasselbe sind, aber doch miteinander in enger Verbindung stehen. Die Sprache bestimmt eigentlich die Denkweise, denn das, was wir hören oder lesen, in unseren mentalen Strukturen ohne unseren Einfluss wiederholt, also „,innerlich nachgesprochen“ wird (ebd.: 147). ${ }^{3}$ Dieser Prozess ist auch mit der Entstehung von Assoziationen zu verbinden, die bei der Verknüpfung eines bestimmten Wortes oder Ausdrucks mit diesen inneren Prozessen und dem außersprachlichen Kontext entstehen.

\footnotetext{
${ }^{2}$ Dieser Begriff wurde von Derrida geprägt und bezeichnet, vereinfacht gesagt, eine männliche Denkweise (cf. Cornejo/Wozonig 2008: 161).

${ }^{3}$ Dieser Ansatz ist auch in der Theorie der verbalen Mediation vertreten, deren Hauptvertreter Bousfield ist (cf. Bousfield 1961: 81-91).
} 


\section{Die Konstruktion der „Outsider“" in einer Gesellschaft}

Geht es um die Konstruktion der Outsider-Gruppe, ist zugleich die Rede von einer Subordination. Den Outsidern wird in einem gesellschaftlichen Kontext die Gleichberechtigung abgesprochen, und das passiert am häufigsten auf der rassistischen oder sexuellen Basis. Identifizierung einer Person oder einer Gruppe als ,,andere“ muss theoretisch gesehen nicht zugleich diskriminierend sein, aber praktisch sieht das etwas anders aus - derjenige, der kategorisiert, hat oft die Position, d. h. die Macht, kategorisieren zu können, und diese Kategorisierung aus der Machtposition beinhaltet schon an sich selbst eine Strategie, die Van Dijk (1997) als ,positive self-presentation“ beschreibt. Die positive Selbstpräsentation schließt implizit die Kennzeichnung „im Unterschied zu“ ein, was bedeutet, dass dadurch automatisch darauf hingewiesen wird, dass der andere negativ zu beschreiben ist. Die Menschen sind nämlich sozialisiert zu glauben, dass das Unterschiedliche zugleich das Suspekte ist, wodurch viele vollkommen unkritisch alle ihnen sublim und subtil aufgetischten Werte bzw. Vorurteile akzeptieren, ohne sich überhaupt zu fragen, was genau sie an den anderen dermaßen stört, dass sie ihnen die zivilen Grundrechte absprechen wollen, und ohne sich irgendwann zu fragen, was sie damit überhaupt zu erzielen hoffen - die Outsider sind immer noch da, sie leben weiterhin unter den „Normalen".

Das Formen der Outsider hängt eng mit der menschlichen Tendenz zusammen, alles zu klassifizieren. Mit Worten von Wierzbicka (2005: 24) sind die Menschen nicht mehr als ,klassifizierende Tiere“, „,(..) they categorize both the ,content of the world" and events into categories and put labels on them". Dieses Klassifizieren, Kategorisieren bzw. im Kontext dieser Arbeit Polarisieren hat auch eine wichtige gesellschaftliche Funktion, nämlich indem man „das Fremde“" ausschließt, um das ,eigene“ zu beschützen, kontrolliert man den sozialen Raum. Die Macht, die man durch diese Kontrolle bekommt, nutzt man zur Legitimation der Strategien, die man bei der Ausschließung gebraucht.

Dabei geschieht es, dass die dominante, hegemoniale gesellschaftliche Gruppe (in unserem Fall die Heterosexuellen) sich selbst aufgrund einer Tradition die Eigenschaft der Natürlichkeit und des Normalseins zuschreibt, und all die anderen, die von den Standards dieser Gruppe abweichen, automatisch mit der Charakteristik „unnatürlich“ oder „,nicht normal“ versehen werden. Wenn etwas oder jemand als ,unnatürlich“ bezeichnet wird, dann ist es auch nicht zu erwarten, dass er/es durch das Gesetz geschützt wird. Im Kontext der sexuellen Orientierung sieht man das in der Abwesenheit der meisten Zivilrechte (Ehe, Kinderadoption, Erbschaft nach dem Tod des Partners, Sozialversicherung, manchmal auch Jobs im öffentlichen Bereich usw.). Dies könnte man eine legalisierte Diskriminierung nennen, die dann im alltäglichen Leben eine quasi legale Basis für die Ausübung unterschiedlicher Attacken verbaler oder nicht verbaler Art auf die Angehörigen dieser Outsider-Gruppe darstellt.

Bei der hegemonialen Gruppe kann man auch immer eine gewisse Neigung zur Homogenität feststellen, und zugleich, wie schon gesagt, operiert diese Gruppe mit den Begriffen „,natürlich“ und „unnatürlich“, wobei sie ihrer eigenen Seite die Eigenschaft der Natürlichkeit zuschreibt.

Laut Bourdieu (1990: 102) sind im gesellschaftlichen Sinne diejenigen Differenzierungen am effektivsten, die auf scheinbar objektiven Unterschieden beruhen, wie z. B. auf dem natürlichen 
Unterschied zwischen Mann und Frau. Solche Denkweise ist dermaßen tief in die Köpfe eingraviert, dass soziale Konstruktionen als Natur erscheinen. ${ }^{4}$ Dieses ist jedoch in sich selbst kontradiktorisch, denn die Natur ist alles, nur nicht homogen. Wenn jemand durch biologische Gegebenheiten homosexuell ist, und man ihn mit allen Mitteln dazu bringen will, sich dem Überwiegenden in einer Gesellschaft anzupassen (möglicherweise durch konversionstherapeutische Maßnahmen) und sich so zu ändern, dass er wie die Mehrheit wird, dann handelt man eigentlich gegen die Natur.

\section{Diskriminierung und Hasssprache}

Hasssprache hat natürlich mit Diskriminierung zu tun. Absurd ist, dass die Diskriminierung per Gesetz verboten ist und sich als unerwünschte Erscheinung in der Verfassung jedes entwickelten Staates befindet, weil sie nämlich gegen soziale und kulturelle Werte verstößt und zugleich aus eben diesen Werten herauskommt. Diskriminierung ernährt sich von einem bestimmten Wertesystem, das fast unsichtbar gelernt und geerbt wird.

Wie kommt man aber dazu, Sprache mit Gewalt zu verbinden? Wie bereits erörtert, kann man mit manchen sprachlichen Äußerungen einerseits Handlungen ausführen und andererseits kann man Sprache als Waffe benutzen, um einem Schaden zuzufügen, z. B. durch das Beleidigen. In diesem Sinne erklärt Butler:

Wenn wir sagen, daß eine Beleidigung wie ein Schlag trifft, implizieren wir, daß solches Sprechen unseren Körper verletzt. Und das tut es - aber nicht in der gleichen Weise wie durch eine rein körperliche Verletzung. Genauso wie eine körperliche Verletzung auch die Psyche betrifft, so wirkt eine psychische Verletzung auf die körperliche Doxa, jenen gelebten und in der Materialität des Körpers gespeicherten Glauben, der die gesellschaftliche Wirklichkeit konstituiert.

(Butler 2006: 248)

Durch Sprache kann man daher nicht nur zur psychischen Verletzung beitragen, sondern ein Sprachgebrauch kann selbst als eine Form der Gewaltausübung betrachtet werden - man kann durch Sprache jemandem die Würde nehmen und eine Person zu einem dehumanisierten Objekt machen (Schwarz-Friesel 2013: 146), was man durch die Hasssprache als einer der häufigsten Formen der sprachlichen Gewalt auch tut.

Was die Definitionen von Hasssprache betrifft, kann man nicht sagen, dass sich die Forscher darin einig sind, was man genau als Hasssprache bezeichnet bzw. auf welche sozialen Gruppen sich die Hasssprache beziehen kann. Bei vielen Autoren (z. B. Tsesis 2002; Haiman 1993) findet man die Einstellung, dass es sich dabei um unsoziale und pejorative Verbalisierungen handelt, die sich auf Rasse, Religion, Nationalität oder eine bestimmte ethnische Gruppe beziehen (cf. Lillian 2007: 731). Beispielsweise bezieht sich Meibauer (2013: 1) in seinen Überlegungen über Hassrede auch überwiegend auf Bevölkerungsgruppen (im Sinne der Nationalität) und definiert die Hassrede als ,den sprachlichen Ausdruck von Hass gegen Personen oder Gruppen, der der Herabsetzung oder Verunglimpfung“ dient. Die sexuelle Orientierung oder Geschlecht überhaupt wird als Ziel der verbalen Attacke in solchen Definitionen häufig nicht erwähnt,

\footnotetext{
${ }^{4}$ Beispielsweise ist die Nation auch ein unnatürliches Konstrukt, mit dem man in einem Punkt der Geschichte künstliche Grenzen um die Menschen herumgestellt hat; gesellschaftliche Normen, die Gleichheit unter unterschiedlichsten Persönlichkeiten verlangen, kann man auch als unnatürlich bzw. künstlich betrachten.
} 
möglicherweise auch deswegen, weil man generell die Einstellung hat, dass die Geschlechtszweiteilung in Mann und Frau eigentlich unfraglich ist. In der neueren Literatur gibt es aber schon Überlegungen, die die sexuelle Orientierung in solche Definitionen einbeziehen. So sagt Reddy (2002, in: Lillian 2007: 732): ,(..) hate speech (...) is seen as a discourse of power, dominance and control which is not merely a form of patriarchal oppression, but a kind of performative communication that produces a discourse about homosexuals in order to misrecognize them." Jedoch haben sich die meisten Arbeiten entweder mit sprachphilosophischen oder juristischen Aspekten der Hasssprache befasst (cf. dazu Schwarz-Friesel 2013: 146).

Wenn man von den Überlegungen von Judith Butler (2006: 250) ausgeht, die die Hasssprache als ,die Art von Handlung, die diejenigen zum Schweigen bringen soll, an den sie sich richtet“ beschreibt, so kann man annehmen, dass sich die Hasssprache nicht nur durch Sprache selbst realisieren lässt, sondern dass man sie auch durch andere Handlungen ausführen kann, beispielsweise durch Bilder. ${ }^{5}$

Dass Hasssprache nicht (immer) von den Worten abhängt, hat auch Meibauer (2013: 1) bemerkt und gesagt, „dass Hass auch nicht-verbal ausgedrückt werden kann, z. B. durch (...) Bilder“, wie es in dieser Arbeit an einigen Beispielen gezeigt wird. Dabei geht es darum, dass man aufgrund der eigenen Zugehörigkeit zu einer bestimmten Gruppe über dieselben oder ähnlichen Bilder verfügt, die man in dieser Gruppe hinsichtlich einer anderen Gruppe vorfinden kann. Im Kontext der Hasssprache geht es dabei um die Feindbilder, die man als Gesamtheit der Eigenschaften, die die fremde Gruppe zur fremden Gruppe macht, definieren kann. Diese Feindbilder befinden sich im kollektiven Bewusstsein der eigenen Gruppe, sind aber auf keinen Fall einfache Abbildungen des Feindes, denn das würde implizieren, dass es den Feind zuerst gegeben hat und dass die Feindbilder aufgrund der Erfahrung mit dem Feind entstanden sind. Die Bilder, die nicht auf Erfahrungen beruhen (und beim Hass gegen soziale Gruppen ist es häufig der Fall), sind einfach die Bilder, wie Angehörige unterschiedlicher ,,sozialen Gruppen einander sehen wollen. Bilder strukturieren Erfahrungen vor, was soweit gehen kann, dass sie Menschen daran hindern, einander anders wahrzunehmen als das Bild, das sie von der sozialen Gruppe haben, zu der sie gehören“" (Haubl 2007: 42).

Wenn man sich mit der Hasssprache aus einer kognitivistischen Sicht befassen will, darf man die emotionale Komponente dabei keinesfalls vernachlässigen. Hass ist eine intensive negative Emotion, die sich als ,heftige Abneigung gegenüber einer Person oder Gruppe manifestiert und die oft mit Verbitterung, Wut und als Ohnmacht empfundener Angst" einhergeht (cf. Schwarz-Friesel 2013: 148). Wenn eine solche Emotion verbalisiert und in diesem verbalen Prozess gegen eine konkrete Person oder Gruppe ausgerichtet wird, bilden sich im mentalen Bereich Bilder bzw. entstehen Konzeptualisierungen, die ein hohes Aggressionspotenzial haben. Außerdem hängt die Abneigung, die man bei der Emotion Hass spürt, eng mit dem Ekel zusammen, der sich als eines der physisch bedingten Hauptgefühle bei Menschen entwickelt. Durch die Hasssprache, besonders im Zusammenhang mit den Homosexuellen, werden eben

\footnotetext{
${ }^{5}$ Zugleich sieht sie aber eine Verdoppelung der performativen Kraft der verbalen Äußerung, die sich als Hasssprache klassifizieren lässt, und zwar in dem Moment, in dem der zum Schweigen Gebrachte, also die Zielperson der verbalen Attacken, dieselben Worte aufgreift und sie in einer Antwort auf den Angriff benutzt. In diesem Prozess verlieren die Worte ihre ursprüngliche Autorität und lassen sich performativ auch gegen den Angreifer richten (ebd.).
} 
die kognitiven Frames des Ekels geweckt, was zu einer konzeptuellen Entmenschlichung ${ }^{6}$ dieser sozialen Gruppe führt, wodurch ihr jeglicher sozialer Wert abgesprochen wird. Dadurch wird auch der Raum für nicht nur verbale Gewalt gegen diese Gruppe geschaffen.

\section{Das kroatische Beispiel oder Was hat die katholische Kirche mit Sex zu tun?}

Das oben Gesagte konnte man ganz klar am Beispiel eines kroatischen Referendums betrachten, an dem man sich für eine Definition der Ehe als Partnerschaft zwischen Mann und Frau bzw. für oder gegen das homosexuelle Recht auf Ehe deklarieren sollte.

In diesem Fall muss gleich eine starke Beziehung zwischen Sprache und Macht auffallen, da es sich dabei, strenggenommen, nur um eine Definition gehandelt hat. Die Art und Weise, auf die man die Institution der Ehe definiert, hat nationale, religiöse, politische und intellektuelle Geister mehr aufgewühlt als beispielsweise die Tatsache, dass tausende von Menschen in den letzten Jahren ihre Arbeit verloren haben und hunderte von ihnen zu Obdachlosen geworden sind. Dass diese Definition, also nur das Sprachliche, große Konsequenzen im realen Leben der Menschen haben kann, steht hier außer Frage. Wer also die Definitionsmacht besitzt, kann einschließen und ausschließen, wie er will bzw. ihm gehört das Recht, die Kriterien zu bestimmen, die eine Person als tauglich oder nicht für eine gesellschaftliche, ja sogar menschliche Funktion (z. B. Eltern zu werden) beschreiben.

Der Ehe als einer institutionalisierten Partnerschaft zwischen Mann und Frau wird ein Selbstverständlichkeitscharakter zugeschrieben, und die Homosexuellen bedrohen dieses Selbstverständliche an der Ehedefinition. Die dominante Gruppe, die die Definitionsmacht behalten wollte, hatte sowohl dies als auch etwas Anderes befürchtet - den Verlust der Ausschließlichkeit und Einzigartigkeit, den Verlust der Macht in der Gesellschaft. Da man aber nicht so offen sagen konnte, dass man sich vor dem Verlust der Macht fürchtet, musste man andere Strategien finden, um den Anspruch auf die Definitionsmacht rechtfertigen zu können. Die Kinder und ihre Rechte waren einfach wie geschaffen dafür, dass man auch diejenigen, die nicht viel von der Institution der Ehe halten, zum Nachdenken bringt, ob man mit dem Erlauben der homosexuellen Ehe doch nicht die Kinder im Stich lässt und sie etwas Unbekanntem, somit auch potenziell Schlechtem und Gefährlichem aussetzt. Auf einmal hatte man die Kinderrechte diskutiert, und die Frage der Definition der Ehe hat man kaum mehr erwähnt. So hat man sublim zwei Strömungen geschaffen - diejenige, die für die Kinderrechte und diejenige, die dagegen war.

Die katholische Kirche spielte in dieser Diskussion eine große Rolle. Es deklarieren sich nämlich mehr als $70 \%$ der Kroaten als Angehörige der katholischen Kirche, und es ist nicht unbekannt, dass die kroatische Gesellschaft im Allgemeinen eine ziemlich traditionelle und patriarchale ist. Diese Tatsache haben die Geistlichen ausgenutzt, sowohl an den Messen von den Altären als auch in den Medien das Volk zum Schutz der Kinder vor dieser unbekannten schwulen Gefahr aufzurufen. Das Hauptthema der in den öffentlichen Medien präsentierten Diskussion war, was für Konsequenzen Geschlechtsverkehr zwischen zwei Männern oder zwei Frauen

\footnotetext{
${ }^{6}$ Haubl (2007: 9) hat dazu gesagt: „Die Menschen, welche gehasst werden, werden als Hassobjekte gekennzeichnet, und nicht nur weil man so sagt, sondern man sagt es so, weil jeder Hass darauf aus ist, anderen Menschen ihren Status als Subjekt zu nehmen.“
} 
auf potenzielle Kinder in dieser Familie haben könnte. Dabei hat man sich natürlich auch auf die Heiligkeit der Ehe berufen. ${ }^{7}$

Im Kontext der öffentlichen Diskussion über die Homosexualität, ihrer Natürlichkeit, darüber, ob diese als Krankheit, Deviation oder eine natürliche Eigenschaft betrachtet werden soll, wurde auch die Frage der Pädophilie aufgeworfen. Egal ob man Homosexualität mit der Pädophilie verglichen hat oder ob man entschlossen war, dass diese zwei, nennen wir sie so, Erscheinungen, nichts miteinander zu tun haben, war der Schaden schon angerichtet - denn laut Lakoff (2004: 3), auch wenn man einen Rahmen negiert, ruft man ihn dadurch herbei. Somit ist die Verbindung zwischen Homosexualität und Pädophilie hergestellt worden, und in den mentalen Konstruktionen vieler Menschen gab es keinen Ausweg mehr.

\section{Strategien der Realisierung der Hasssprache gegen Homosexuelle in Kroatien}

Generell betrachtet, kann man sagen, dass, wenn es auf öffentliche Persönlichkeiten in öffentlichen Auftritten ankommt, die Strategien des Ausdrucks und der Verbreitung von negativen Assoziationen in Bezug auf Homosexuelle eher einen impliziten Charakter hatten. Die Strategien, die man auf den Seiten unterschiedlicher sozialer Netzwerke wie Facebook oder Twitter finden konnte, waren schon expliziter, gelegentlich auch brutal. Hier bringen wir einige Beispiele aus beiden Kommunikationssphären, wobei wir der Meinung sind, dass die impliziten Hassausdrücke noch gefährlicher sind als die expliziten, denn sie werden als Meinungsfreiheit akzeptiert und geraten auf diese Weise ohne Zensur in die alltägliche Kommunikation ,und können so zum einen den alltäglichen Sprachgebrauch affizieren, zum anderen Einfluss auf das kollektive Bewusstsein der Gesellschaft nehmen“ (Schwarz-Friesel 2013: 156f).

\section{$\underline{\text { Beispiel } 1}$}

Diese Initiative hatte einen moralischen Charakter (...) und es war selbstverständlich, dass die Kirche sie unterstützt hat. ${ }^{8}$

Im oben angeführten Beispiel sieht man eine Strategie, die man als Argumentierung eines berechtigten Vorgehens beschreiben kann. Vom Hass ist auf den ersten Blick keine Rede, und es gibt auch keine explizite verbale Beleidigung in den Worten, die ein kroatischer Bischof an die Priester, Gläubigen und alle anderen Bürger Kroatiens gerichtet hat. Es war selbstverständlich - in diesem Teil der Aussage liegt der Großteil des Problems. Dieser Aussage nach, scheint es für die Kirche selbstverständlich, dass eine gesellschaftliche Gruppe marginalisiert ist, als wäre es selbstverständlich, sich in das Sexuelle und in das Staatliche im Sinne der Zivilrechte einzumischen. Diese Selbstverständlichkeit ist deshalb gefährlich, weil man schwer gegen sie kämpfen kann; wenn etwas selbstverständlich ist, dann braucht es keine Argumentation. Oder mit

\footnotetext{
7 Was von der Forderung auf Anerkennung der homosexuellen Ehe Bourdieu zu sagen hat, kann man in den folgenden Zeilen lesen: „Gleichzeitig ist diese Forderung, eben weil man - und wieder findet sich der Katholizismus ein - die Ehe zu einer derart „heiligen“ Angelegenheit macht, ausgestattet mit höchster Symbolkraft, dazu geeignet, wie nichts anderes unsere Vorstellungen, die überlieferten Denkweisen zu sprengen. Ich meine, daß diese Art der symbolischen Herrschaftsausübung die perverseste Form der Herrschaft überhaupt ist. Wenn man das alles zusammennimmt, dann bedeutet die Anerkennung einer ,homosexuellen Ehe“: Abschaffung von Diskriminierungen und die Öffnung der Gesellschaft für wichtige Fragen, nicht nur über die Institution der Ehe selbst, sondern über unsere moralische Ordnung überhaupt" (Bourdieu 2001:26).

8 Aus dem Artikel auf der Webseite: http://narod.hr/clanak/biskup-bogovic-jasno-je-da-podrzavam-izjasnjavanjeza-referendum-preporucujem-je-svim-svecenicima-vjernicima-svim-građanima [15. 10. 2015].
} 
Worten von Schwarz-Friesel: der implizite Hass wird als Sprachhandlung rekodiert, die formal entradikalisiert ist, inhaltlich jedoch die gleichen Merkmale aufweist wie die Hassrede von Extremisten (2013: 160).

Das zweite Problem, das man in einer solchen Aussage sieht, liegt im Wort moralisch. Den Homosexuellen die Ehe zu verbieten, hängt mit Moral zusammen, sagte der Bischof, weil das auch selbstverständlich war. Mit anderen Worten hat er den Homosexuellen die Moral abgesprochen, er hat sie als unmoralische Wesen etikettiert, und wenn jemand von einer Machtposition spricht und jemanden als unmoralisch bezeichnet, dann gibt er den anderen die Erlaubnis, sich diesem gegenüber ohne jegliche Rücksicht zu verhalten. Die Machtposition von der der Bischof sprach, bezieht sich nicht nur auf seine Position in der Kirche, sondern auf die Kirche im Allgemeinen. Er selbst hat sich der Methode der Entpersonalisierung bzw. der Meinungsgeneralisierung bedient, denn er sprach nicht in seinem eigenen Namen, sondern im Namen der ganzen katholischen Kirche. In dieser Aussage haben viele Gläubige die Rechtfertigung für das weitere Hetzen gegen die Homosexuellen gefunden, denn sie haben sich als Wächter der öffentlichen Moral gefühlt. Wie man sieht, kann der Hass auch in ruhige und quasi höfliche Meinungsäußerungen verpackt werden, was einem ermöglicht, ungestört auf die Meinungsbildung anderer Menschen zu wirken.

\section{Beispiel 2}

\section{Wir haben gesiegt, genauso wie David den Goliath besiegt hat.}

Der Vergleich, der in diesem Beispiel ausgedrückt ist, versteckt unterschiedliche konzeptuelle Verbindungen und Konnotationen. Zuerst geht es hier um die Ungleichheit der Gegner, wo die Homosexuellen mit Goliath gleichgesetzt werden. Der Kampf zwischen David und Goliath ist ein Synonym für eine ungerechte Verteilung der Macht und dafür, dass alles möglich ist, wenn man in Gottes Namen handelt. Die Organisationen, die den Homosexuellen die Institution der Ehe verbieten wollten, haben sich selbst die Eigenschaften des Davids zugeschrieben - also die schwächere Machtposition in der Gesellschaft und den Glauben bzw. das Handeln nach Gottes Willen. Zugleich bedeutet es, dass die Homosexuellen übermächtig sind, dass sie nicht an Gott glauben und dass Gott auf keinen Fall an ihrer Seite stehen kann. Wenn man die Situation auf diese Weise betrachtet, wird klar, dass der Goliath nur negative Konnotationen hervorrufen kann, und mehr noch: er war auch unnatürlich, weil er mehr als drei Meter groß war. Jemand, der so groß ist, stellt eine Bedrohung dar. Diese triumphierende Aussage impliziert also, dass die Homosexuellen eine Bedrohung für die Gesellschaft darstellen, und diese Bedrohung gehört beseitigt, was mit dem abgehaltenen Referendum erzielt werden wollte. Nur, die Tatsache ist, dass sie nicht beseitigt wurden, sondern dass ihnen damit ein Zivilrecht abgesprochen wurde.

\section{$\underline{\text { Beispiel } 3}$}

Genauso wie man in einer nicht weit zurückliegenden Vergangenheit die Homosexualität von der Liste der Deviationen gestrichen hat, passiert dasselbe jetzt auch mit Pädophilie. ${ }^{9}$

Mit diesem Beispiel wollten wir zeigen, wie man konzeptuelle Verbindungen schafft, durch die eine soziale Gruppe stetig als Feind etikettiert wird. Die Kinder gelten als einer der höchsten

\footnotetext{
${ }^{9}$ Aus dem Artikel auf der Webseite: http://zdravstveniodgoj.com/news/pedofilija-je-kao-i-homoseksualnost-postala-seksualna-orijentacija (veröffentlicht am 1. 11. 2013) [17. 10. 2015].
} 
Werte in allen entwickelten Gesellschaften. Derjenige, der den Kindern schadet, verdient nichts Gutes und soll möglichst streng bestraft werden - Kinder sind unschuldig und hilflos, und es ist die Aufgabe der Erwachsenen, sie zu beschützen. Das Problem der Pädophilie ist so schrecklich, dass man sich dem gegenüber fast wie einem Tabu gegenüber verhält. Viele Eltern würden nicht zögern, denjenigen, der sich an ihrem Kind in dem Sinne ergreift, zu töten oder zum Tode zu verurteilen. Also verkürzt: Pädophilen werden in einer Gesellschaft als das größte Übel behandelt, das ausgerottet gehört. Kommen wir nun zu unserem Beispiel zurück, in dem mit einem solchen Übel die Homosexuellen verglichen werden bzw. sie werden in denselben Kontext gestellt. Zwischen den Zeilen lesend, heißt es, dank der Tatsache, dass Homosexualität als sexuelle Orientierung und keine Störung behandelt wird, passiert dasselbe jetzt mit der Pädophilie. Man gibt einerseits den Homosexuellen die Schuld dafür, und andererseits gleicht man diese zwei Erscheinungen aus (weil sie einst auch ausgeglichen waren, also bevor die Homosexualität zur sexuellen Orientierung erklärt wurde). Je öfter man zwei Ideen im selben Kontext erwähnt, desto mehr glauben die Leute, dass sie wirklich verbunden sind, denn die Neurowissenschaftler haben bewiesen, dass ,,je häufiger eine Verknüpfung aktiviert wird, desto stärker wird die neuronale Verbindung zweier Ideen“ (Lakoff/Wehling 2008: 18). Wenn eine Idee also ins Gehirn inkorporiert wird, dann ist man kaum in der Lage, anders zu denken, denn ungefähr $80 \%$ unseres Denkens läuft vollkommen unabhängig von uns ab bzw. ist uns nicht bewusst (Lakoff/Wehling 2008: 13).

Beispiel 4

\section{THIS IS NOT HATRED:

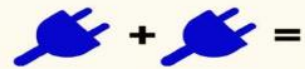$$
\text { (a) }+0
$$$$
3+C=
$$ \\ IT'S BALIED SBIENBE.}

Abbildung 1: Berufung auf Wissenschaft als Rechtfertigung der Hassrede

In der Kampagne gegen die Homosexuellen hat man sich in den öffentlichen Interviews sehr häufig auf einige wissenschaftliche Untersuchungen berufen, laut welchen die Kinder, die in einer homosexuellen Partnerschaft aufwachsen, schwächere Leistungen in der Schule erbringen und im erwachsenen Alter mehr zu Suizid neigen als die gleichaltrigen, deren Eltern heterosexuell sind. Diese Untersuchungen haben sich irgendwann bald nach ihrer Entstehung entweder als nicht wissenschaftlich oder verfälscht erwiesen, aber da sie zugänglich waren, hat man sich stark an ihnen orientiert. Also war die Strategie der Selbstaufwertung und Legitimierung durch Wissenschaftliches sehr präsent und sehr wirksam, weil sich viele von denjenigen, die vielleicht noch unentschieden waren, ausgerechnet deswegen ,dafür" (für die vorgeschlagene Ehedefinition) entschieden haben. Sie wollten nämlich nicht dazu beitragen, dass irgendein Kind gleich am Anfang seines Lebens benachteiligt wird, wie in solchen Aussagen präsentiert wurde. Natürlich hat man auch danach gewusst, dass die Resultate dieser Untersuchungen nicht gestimmt haben, aber man wollte nichts dem Zufall überlassen, oder wie Bourdieu (1990: 109) es gesagt hat, die Macht eines kategorischen attributiven Urteils, der durch die Institutionalisierung zustande kommt, sei so groß, dass keine komplett entgegensetzte Realisierung in der Praxis es

ISSN 1615-3014 
aufheben könne. Indikativ ist für dieses Beispiel auch die Tatsache, dass die wissenschaftlichen Beweise, auf die man sich in der Argumentation der eigenen Position berufen hat, von den amerikanischen Wissenschaftlern stammten - dies sollte auch als kein Zufall betrachtet werden, denn in einem kleinen und nicht ganz entwickelten Land, hat alles Westliche, besonders alles Deutsche und Amerikanische, eine viel größere Wirkung als das Kroatische. Daher war es selbstverständlich, dass man den Untersuchungen von amerikanischen Wissenschaftlern Glauben geschenkt hat, obwohl viele kroatische Wissenschaftler öffentlich gegen solche Resultate argumentiert haben. Mit dem bildlichen Teil in diesem Beispiel wollte man zeigen, dass die homosexuelle Partnerschaft unproduktiv ist, dass aus ihr nichts entsteht und sie keinen Sinn hat, sowie dass diese eigentlich nach den Gesetzen der Evolution nicht überleben sollte.

\section{$\underline{\text { Beispiel } 5}$}

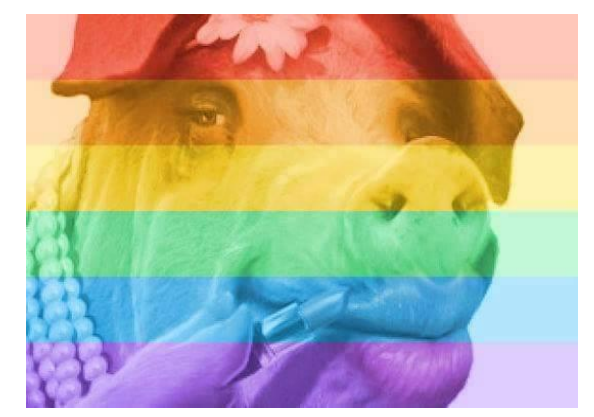

Abbildung 2: Gleichsetzung der Homosexuellen und Schweine

Die bekannte Aussage Tucholskys lautet: ein Bild sagt mehr als tausend Worte, und an diesem Beispiel sieht man sehr klar, wie man ohne irgendwelche Worte den Hass ausdrücken kann. Die Strategie der Entmenschlichung stellt bei der Bildung der Basis für sprachliche (und nicht nur sprachliche!) Gewalt eine beliebte Vorgehensweise dar. Diese Methode ist auch sehr effektiv, weil durch sie eine quasi Erlaubnis erteilt wird, die durch gesellschaftliche Normen bestimmte und mittlerweile als immanent empfundene Zurückhaltung und Höflichkeit zu vergessen. Damit wird jedes Mittel gerechtfertigt - wir kämpfen gegen das Unmenschliche, gegen das Unnatürliche (ein Schwein mit Lippenstift, Blume und Halskette, mit einer Karikatur der menschlichen Lippen und Augen). Wir gegen sie - diese zwei Gruppen, die die Verteilung der Machtpositionen in der Gesellschaft widerspiegeln, weisen darauf hin, dass weder derjenige, der hasst noch derjenige, der gehasst wird ein Individuum darstellt. Es geht dabei um den kollektiven Hass, der gegen eine Gruppe gerichtet ist. Unausgesprochen, aber im kollektiven Bewusstsein präsent, verbindet man Schweine mit Schmutz und Krankheiten (in der menschlichen Geschichte haben Schweine schwere Krankheiten übertragen), und egal wie unschuldig ein solches Bild auf den ersten Blick aussehen mag, versteckt es doch Konnotationen, die auf keinen Fall harmlos sind. Dieses Bild ist kein Zufall, es ist ein Feindbild - man könnte sich als Probe einen Schwan anstelle des Schweins vorstellen und man würde bestimmt einsehen, dass der Effekt gar nicht der gleiche ist. Dieses Beispiel ist auch ein guter Beweis dafür, dass die Basis der Feindbilder nicht unbedingt die Erfahrung ist, sondern eher die Vorstellungen, die man von jemandem hat. 


\section{$\underline{\text { Beispiel } 6}$}

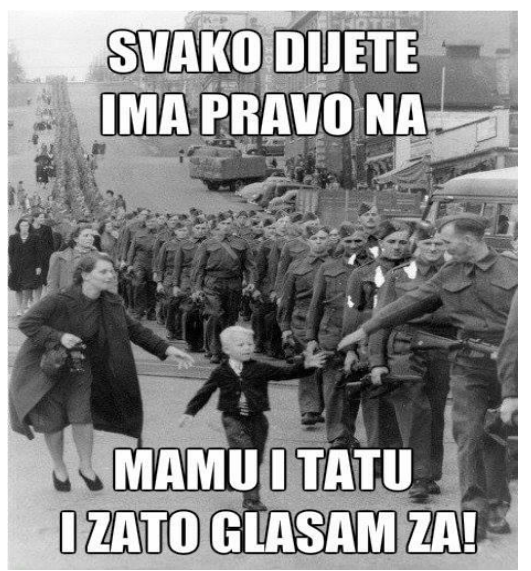

Abbildung 3: Jedes Kind hat das Recht auf Mutti und Vati. Deswegen bin ich „dafür“

Die Strategie der Ablenkung vom wahren Problem - so könnte man das in diesem Beispiel beinhaltete Manöver beschreiben. Hier bezieht man sich nicht expressis verbis auf Homosexuelle, sondern sie werden implizit, und zwar über den Referenzaspekt „Kinderrechte“ eingeführt. Wie also schon vorhin angedeutet, wurde die Kampagne gegen die homosexuelle Ehe an eine andere „Front“" versetzt, d. h. man hat sich sehr bald nach dem Anfang der Kampagne auf das Verletzen der Kinderrechte orientiert, womit das Resultat eigentlich im Voraus bestimmt war. Die Erlaubnis, eine homosexuelle Ehe zu schließen, würde nämlich bedeuten, dass homosexuelle Paare auch Kinder adoptieren könnten, und dieser Gedanke wurde in der kroatischen Gesellschaft nicht so gut angenommen, sogar bei vielen Hochgebildeten stieß er auf Ablehnung. Die Frage der Kinderrechte hat die Gruppe, die für die schon erwähnte Definition der Ehe war, noch homogener gemacht. Bei solchen Polarisierungen auf Wir- und Sie-Seite gilt im Allgemeinen, dass das schwächste Mitglied der Wir-Seite immer noch besser als das beste Mitglied der Sie-Seite ist. Man hat also nicht überlegt, wie viele Kinder, die heterosexuellen Ehen entstammen, sich in diversen Erziehungsanstalten befinden und wie viele immer noch mit ihren Eltern wohnen und ein sehr schlechtes Leben haben - das war vollkommen irrelevant. Hauptsache war, dass man dem Kind das Recht auf Mutter und Vater nicht nehmen darf. Wenn man sich das oben präsentierte Bild anschaut, fällt einem sofort die Aufrufung des kollektiven Bewusstseins auf, das bei den Kroaten besonders leicht durch das Militärische aktiviert wird, und zwar aufgrund des Krieges in den 1990er Jahren. Das Kriegerische erinnert an das Zusammenhalten in schwierigen Zeiten und daran, dass man gewinnt, wenn man zusammen kämpft. So muss man auch in diesem Kampf gegen die Homosexuellen vorgehen, denn Kinder und ihre Rechte gehören geschützt. Das Foto stammt wahrscheinlich aus dem Zweiten Weltkrieg, wodurch an Tradition erinnert wird, und traditionelle Werte in einer traditionellen Gesellschaft, wie es die kroatische ist, haben immer den Vorrang. Unausgesprochen und sehr, sehr implizit, wird hier auch auf das Nationale abgehoben, denn das ist ein Aspekt, wogegen die Kroaten immer noch sehr empfindlich sind. Also ist die Strategie in diesem Beispiel die folgende: Man weckt das nationale Gefühl, das für die Homogenität der Gruppe sorgt und sie festigt, dann nimmt man die Kinder als die hilflose und unschuldige soziale Gruppe, die man beschützen soll, und richtet die erneut entstandene Einigkeit gegen ein beliebiges Problem, in unserem Fall, gegen die Homosexuellen. Erfolg ist garantiert. 


\section{Schlussbemerkungen}

Den oben angeführten Beispielen lässt sich entnehmen, wie man mentale Bilder mithilfe von Sprache konstruiert und wie sich diese mentalen Bilder leicht in hassvolle Abbildungen übertragen lassen. Um hassvolle Bilder sowie hassvolle Sprache produzieren zu können, muss man durch sie auf jemanden referieren. Die Referenten solcher durch Negativität geladenen Bilder und Worte entstehen durch die Einteilung der Gesellschaft, die auf gewissen sowohl realen als auch fiktiven Unterschieden beruht. In der in dieser Arbeit untersuchten Problematik geht es darum, dass sich Homosexuelle durch ihre sexuellen Präferenzen von den Heterosexuellen unterscheiden, wobei wir von einem realen Unterschied reden können. Da dieser Unterschied selbst als kein ausreichendes Argument für die Entstehung einer negativ gekennzeichneten Polarisierung in der Gesellschaft dienen kann, musste man noch zahlreiche fiktive Unterschiede konstruieren, durch die extrem negative mentale Bilder entstehen konnten. Diese fiktiven Unterschiede dienen erst als Basis für die Äußerungen, die wir als Hassrede beschreiben können. In diesem konkreten Fall wurden die Homosexuellen mit Schmutz, Perversion (Pädophilie), Unnatürlichkeit usw. verbunden und es wurden ihnen überhaupt menschliche Charakteristika abgesprochen, und zwar mit dem Ziel, bei verbaler (oder physischer) Aggression gegen diese gesellschaftliche Gruppe keine Skrupel haben zu müssen.

Wie schon am Anfang dieser Überlegungen betont, erfolgt die Polarisierung der Gesellschaft auf einer symbolischen Ebene, auf der bestimmte negativ geladene Konzepte entstehen, die unbemerkt zur allgemein akzeptierten Meinung werden und als Basis für (nicht nur) sprachliche Gewalt dienen. Bei jeder Art der gesellschaftlichen Polarisierung muss man davon ausgehen, dass es eine hegemoniale Gruppe gibt, die unterschiedliche Charakteristika nach Belieben verteilt. Diese Gruppe operiert aus einer Machtposition und kann ihre eigene Seite als (ausschließlich) positiv, und die andere Seite als (ausschließlich) negativ bezeichnen - wenn solche Kategorisierungen immer wieder wiederholt werden, werden sie allmählich auch akzeptiert. Auf diese Weise entstehen die sog. Wir- und Sie-Seite, wobei die Sie-Seite als Outsider präsentiert wird, woraus die Wir-Seite die Rechtfertigung für ihr Vorgehen gegenüber der Sie-Seite schöpft. Eine solche Zweiteilung der Gesellschaft ist in der oben durchgeführten Analyse sehr deutlich, und aufgrund der dabei offensichtlichen starken Polarisierung konnte man unterschiedliche Strategien in diesem „Kampf gegen Goliath“ verwenden. Vor allem ging es dabei um Selbstaufwertung, Argumentation, (quasi)wissenschaftlicher Legitimierung und Diffamierung. Manchmal wurden diese Strategien durch eher implizite Worte und Bilder realisiert, und manchmal waren sie sehr explizit. Die implizite Vorgehensweise war häufiger in den Printmedien und in den Fernsehsendungen vertreten, wobei man sich auf den Seiten der sozialen Netzwerke und in öffentlichen Diskursen, in denen man sich über die aktuelle Problematik äuBern konnte (Sammlungen, Kirchenpredigten), kaum um die Implizitheit der Aussagen bemüht hat.

\section{Literatur}

Austin, John Langshaw (1979): Zur Theorie der Sprechakte (How to do Things with Words). 2. Auflage. Stuttgart: Reclam.

Bourdieu, Pierre (1990): Was heißt sprechen? Zur Ökonomie des sprachlichen Tauschens. Wien: Braumüller.

Bourdieu, Pierre (1991): Language and Symbolic Power. Cambridge: Polity Press. 
Bourdieu, Pierre (2001): „Teilen und herrschen: Zur symbolischen Ökonomie des Geschlechterverhältnisses“. In: Rademacher, Claudia/Wiechens, Peter (eds.): Geschlecht - Ethnizität-Klasse. Zur sozialen Konstruktion von Hierarchie und Differenz. Opladen, Leske+Budrich: 11-31.

Bousfield, Weston A. (1961): "The problem of meaning in verbal learning". In: Cofer, Charles/ Musgrave, Barbara (eds.): Verbal learning and verbal behavior. New York, McGraw-Hill Book Company: 81-91.

Butler, Judith (1999): Gender Trouble. Feminism and the Subversion of Identity. New York, London: Routledge.

Butler, Judith (2006): Haß spricht. Zur Politik des Performativen. Frankfurt a. M.: Suhrkamp. Cornejo, Renata/Wonzonig, Karin (2008): „Gender Studies in der Literaturwissenschaft - Literaturwissenschaft in den Gender Studies. Eine Wechselwirkung“. In: Thuswaldner, Gredor (ed.): Derrida und danach? Literaturtheoretische Diskurse der Gegenwart. Wiesbaden: VS Verlag für Sozialwissenschaften.

Fauconnier, Gilles/Turner, Mark (2003): The Way We Think. Conceptual Blending and the Mind's Hidden Complexities. New York: Basic Books.

Graumann, Carl Friedrich (1972): „Die Beziehung zwischen Denken und Sprechen als psychologisches Problem“. In: Rücktäschel, Annamaria (ed.): Sprache und Gesellschaft. München, Fink: $139-153$.

Haiman, Franklin (1993): 'Speech acts' and the First Amendment. Carbondale: Southern Illinois University Press.

Haubl, Rolf (2007): „Gattungsschicksal Hass“. In: Haubl, Rolf/Caysa Volker (eds.): Hass und Gewaltbereitschaft. Göttingen, Vandenhoeck \& Ruprecht: 7-68.

Hetzel, Andreas (2010): Die Wirksamkeit der Rede. Zur Aktualität klassischer Rhetorik für die moderne Sprachphilosophie. Bielefeld: transcript.

Jäkel, Olaf (2003): Wie Metaphern Wissen schaffen. Hamburg: Verlag Dr. Kovač.

Lakoff, George/Johnson, Marc (1980): Metaphors we live by. Chicago: The University of Chicago Press.

Lakoff, George (2004): Don't think of an elephant! Know your Values and Frame the Debatte. Vermont: Chelsea Green Publishing.

Lakoff, George/Wehling, Elisabeth (2008): Auf leisen Sohlen ins Gehirn. Politische Sprache und ihre heimliche Macht. Heidelberg: Carl-Auer.

Lillian, Donna L. (2007): "A thorn by any another name: sexist discourse as hate speech". Discourse Society 18: 719-740. http://das.sagepub.com/content/18/6/719 [18. 10. 2015].

Meibauer, Jörg (2013): „Hassrede - von der Sprache zur Politik”. In: ders. (ed.): Linguistische Untersuchungen 6: Hassrede/Hate Speech. Interdisziplinäre Beiträge zu einer aktuellen Diskussion. Gießener Elektronische Bibliothek: 1-17.

Schwarz-Friesel, Monika (2013) „,,Das ist kein Hassbrief - sondern meine eigene Meinung über Euch“ - Zur kognitiven und emotionalen Basis der aktuellen antisemitischen Hassrede.“ In: Meibauer, Jörg (ed.): Linguistische Untersuchungen 6: Hassrede/Hate Speech. Interdisziplinäre Beiträge zu einer aktuellen Diskussion. Gießener Elektronische Bibliothek: 143-164.

TenHouten, Warren D. (2007): A General Theory of Emotions and Social Life. London/New York: Routledge.

Tsesis, Alexander (2002): Destructive Messages: How Hate Speech Paves the Way for Harmful Social Movements. New York: University Press. 
Van Dijk, Teun A. (1997): "Political Discourse and Racism: Describing Others in Western Parliaments." In: Riggins, Stephen Harold (ed.): The Language and Politics of Exclusion: Others in Discourse. London, Sage: 31-64.

Wierzbicka, Anna (2005): Emotion Across Languages and Cultures: Diversity and Universals. Cambridge: Cambridge University Press.

Zingo, Martha (1998): Sex/gender outsiders, hate speech and freedom of expression: Can they say that about me? Connecticut/London: Praeger. 